\title{
The Comparisons of Surgical Outcomes and Clinical Characteristics between the Far Lateral Lumbar Disc Herniations and the Paramedian Lumbar Disc Herniations
}

\author{
Hyun Wook Park, Ki Seok Park, Moon Sun Park, Seong Min Kim, \\ Seung Young Chung, Do Sung Lee \\ Department of Neurosurgery, Eulji University Hospital, College of Medicine, Eulji University, Taejon, Korea
}

\begin{abstract}
Objective: The purpose of this study is to analyze clinical characteristics and surgical outcomes of the far lateral and the paramedian disc herniations.

Methods: The 88 patients who underwent an operation for lumbar disc herniations were reviewed. Visual analogue scale of leg and back pain, occurrence of sensory dysesthesia and motor deficit before and after operations were used to compare the far lateral with the paramedian disc herniations.

Results: Statistically, the far lateral herniations had more severe radicular leg pain and showed more frequent occurrence of sensory dysesthesia than paramedian herniations before operation $(p<0.05)$. In the far lateral herniation group, preoperatively, 15 patients $(75 \%)$ had sensory dysesthesia and among them, 4 patients $(27 \%)$ showed improvement. In the paramedian herniation group, preoperatively, 25 patients (37\%) had sensory dysesthesia and among them, 21 patients (84\%) showed improvement. The degree of improvement in sensory dysesthesia was statistically higher in paramedian herniation group $(p<0.05)$. In the far lateral herniation group, preoperatively, 11 patients $(55 \%)$ had motor deficit and among them, 10 patients (91\%) showed improvement. In the paramedian herniations, preoperatively, 29 patients (43\%) had motor deficit and among them, 25 patients (86\%) showed improvement. The degree of improvement in motor deficit was not statistically significant between groups $(p>0.05)$.

Conclusion: Preoperatively, the far lateral herniations had more severe radicular leg pain and frequent occurrence of sensory dysesthesia. Postoperatively, the sensory dysesthesia was less improved and back pain was more severe in the far lateral herniations.
\end{abstract}

Key Words: Far lateral disc herniation $\cdot$ Paramedian disc herniation $\cdot$ Clinical characteristics $\cdot$ Surgical outcomes

\section{INTRODUCTION}

The majority of lumbosacral radiculopathies are caused by the paramedian lumbar disc herniations. However, the foraminal or extraforaminal far lateral lumbar disc herniations extending into or beyond the foraminal zone have been recognized between $7-12 \%$ of all lumbosacral disc herniations, ${ }^{4,8,13}$. The term 'far lateral' is often used to describe a lumbar disc herniation that compresses the exiting nerve root at the same level, lateral to the neural foramen or further laterally,9) (Fig. 1).

\footnotetext{
- Received: July 17, 2013 - Revised: August 26, 2013

- Accepted: August 28, 2013

Corresponding Author: Ki Seok Park, MD

Department of Neurosurgery, Eulji University Hospital, College of edicine,

Eulji University, 1306 Dunsan-dong, Seo-gu, Daejeon 302-799, Korea

Tel: +82-42-611-3442, Fax: +82-42-611-3444

E-mail: ks3432@eulji.ac.kr
}

The far lateral disc herniations have clinical characteristics which distinguish them from the paramedian disc herniations. Generally, the radicular leg pain is more severe in the far lateral lumbar disc herniation than the paramedian lumbar disc herniation due to direct compression of dorsal root ganglion which is an extraordinarily pain sensitive structure ${ }^{7,8)}$ and the far lateral disc herniations often have an acute onset in their clinical courses ${ }^{13)}$. The far lateral lumbar disc herniations show sequestered disc migrating superolaterally into or beyond the neural foramen, which compresses the superiorly exiting nerve root $^{1)}$. Comparing with the paramedian disc herniations, far lateral disc herniations occur more frequently at upper lumbar levels ${ }^{10)}$ which are frequently associated with additional pathological lesions as combination of the paramedian disc herniation or spinal stenosis at the same level (Fig. 2). Another particularity of far lateral disc herniations is their occurrence in older patients, with a peak incidence in the sixth decades ${ }^{11,13)}$. 

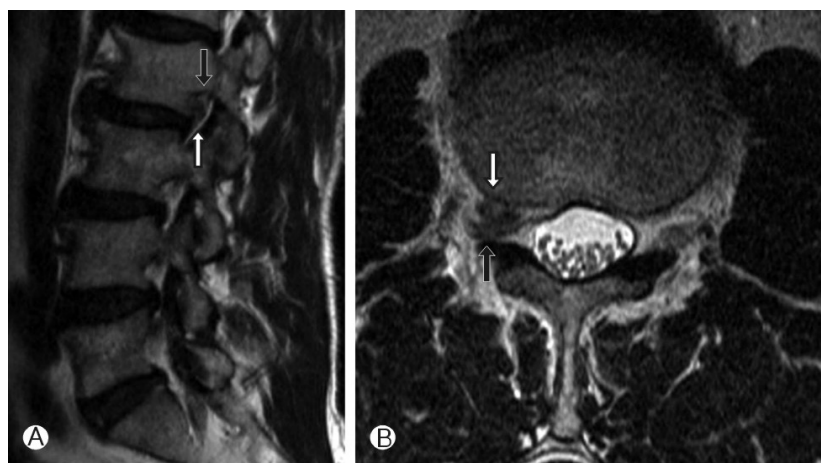

Fig. 1. (A) Sagittal T2-weighted MRI shows the superolateral migrated herniated disc and the compression of superior exiting nerve root at L2-3 level. (B) Transaxial T2-weighted MRI shows the far lateral herniated disc and direct compression of the dorsal root ganglion (white arrowhead: superolateral migrated herniated disc, black arrowhead: dorsal root ganglion).

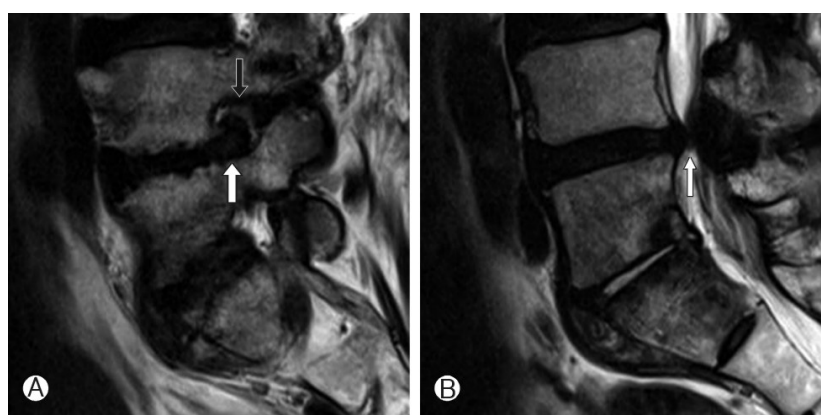

Fig. 2. Far lateral disc herniation associated with additional spinal stenosis (so-called "Double herniations"). (A) T2-weighted MRI shows the superolateral migrated herniated disc at L4-5 level (white arrowhead: superolateral migrated herniated disc). (B) A severe spinal stenosis at the same level (white arrowhead: vertebral canal stenosis).

To our knowledge, there has been no report analyzing about the surgical outcomes and clinical characteristics between the far lateral lumbar disc herniations and the paramedian lumbar disc herniations. In this study, we analyzed surgical outcomes and clinical characteristics between the far lateral lumbar disc herniations and the paramedian lumbar disc herniations.

\section{MATERIALS AND METHODS}

The 88 patients had undergone open discectomies for the far lateral lumbar disc herniations or the paramedian lumbar disc herniations at our institute from January 2008 to January 2013. The data was retrospectively obtained by telephone interviews and reviewing medical charts. The patients were average 52.4 years of age with ranging in age from 22 to

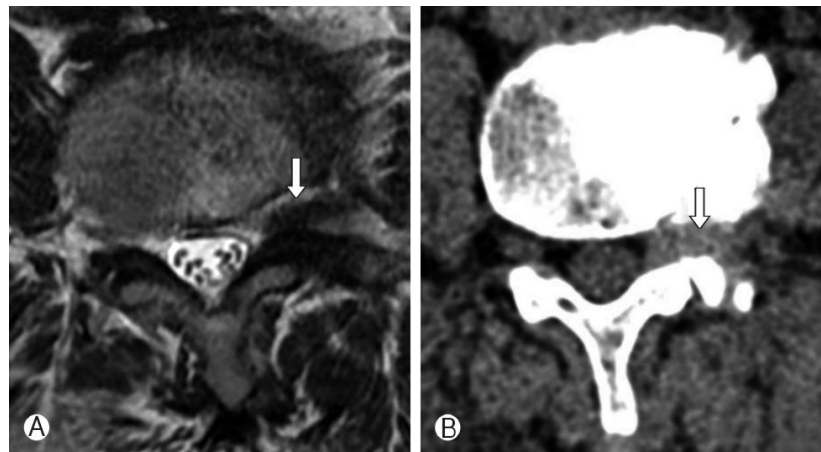

Fig. 3. (A) T2-weighted MRI shows the far lateral disc herniation at L4-L5 level. (B) CT shows the far lateral disc herniation at L4L5 level (white arrowhead: herniated disc).

81 year-old. All patients were assessed by surgeons with telephone interviews. The far lateral disc herniation group included 20 patients and the paramedian disc herniation group included 68 patients. The records of each patient were analyzed with respect to the visual analogue scale (VAS, $0=$ no pain, $10=$ maximum pain) of radicular leg and back pain before and after operation, the occurrence of motor deficit and sensory dysesthesia before operation. Postoperatively, we also analyzed the degree of improvement in the sensory dysesthesia and motor deficit in both groups. The pathology of $88 \mathrm{pa}-$ tients was confirmed by magnetic resonance imaging (MRI) and computed tomography (CT) (Fig. 3). The paramedian approach was used in the far lateral disc herniation group and the conventional midline approach was used in the paramedian disc herniation group.

The paramedian approach used for far lateral disc herniations is performed at the level of the facet joint, provides a more direct route to lesion. In technique of the far lateral disc herniations, we typically performed using $3 \mathrm{~cm}$ lateral to the midline incision and separating the multifidus and longissimus muscle along the natural septa. Transverse process and the lateral facet joint were reached. Lateral aspect of the facet and pars are partially removed followed by transverse ligament is released and herniated disc is removed.

Statistically, Student t-test, Wilcoxon signed ranks test, Paired t-test, Chi-square test were used. The results were considered significant when the probability value (p-value) was less than 0.05. All statistical analyses were performed using commercial software (SPSS 15.0, SPSS Inc., Company, Chicago, IL).

\section{RESULTS}

The patients in the far lateral disc herniation group were older than in the paramedian disc herniation group (61.6 year-old versus 49.6 year-old), which was considered statisti- 
cally significant $(\mathrm{p}<0.05)$. The average follow-up period in the far lateral disc herniation group was 24.3 months and in the paramedian disc herniation group was 32.6 months, but which was not considered statistically significant ( $p>0.05$ ).

The average duration of symptoms before operation was 64.3 days in far lateral disc herniation group and 130.6 days in paramedian disc herniation group, which was considered statistically significant $(\mathrm{p}<0.05)$.

In the far lateral disc herniation group, the operation levels of the 20 patients were L2-L3 in $2(10 \%)$, L3-L4 in $3(15 \%)$, L4-5 in $9(45 \%)$, and L5-S1 in 6 patients (30\%). About 25\% were located at the L2-3 and L3-4 levels. In the paramedian disc herniation group, the operation levels of the 68 patients were L2-L3 in none (0\%), L3-L4 in $2(3 \%), \mathrm{L} 4-5$ in $32(47 \%)$, and L5-S1 in 34 patients (50\%). Only 3\% were located at the L2-3 and L3-L4 levels (Table 1).

The pathology of 20 patients in far lateral disc herniation group showed all extrusion discs from the description on the operation records and among them, 15 patients (75\%) presented with extrusion disc which migrated superolaterally into the neural foramen. They showed the shorter duration of symptoms (29.7 days) before operation than 5 patients (25\%) who presented with extrusion disc without migration (168 days).

The pathology of 68 patients in paramedian disc herniation group could also be divided into protrusion discs and extrusion discs, in 24 (35\%) and 36 cases (53\%), respectively, and sequestration in 8 cases (12\%) from the description on the

Table 1. Distribution of far lateral disc herniations and paramedian disc herniations

\begin{tabular}{lcc}
\hline \hline Level & $\begin{array}{c}\text { Paramedian disc } \\
\text { herniations }\end{array}$ & $\begin{array}{c}\text { Far lateral disc } \\
\text { herniations }\end{array}$ \\
\hline L2-L3 & $0(0 \%)$ & $2(10 \%)$ \\
L3-L4 & $2(3 \%)$ & $3(15 \%)$ \\
L4-L5 & $32(47 \%)$ & $9(45 \%)$ \\
L5-S1 & $34(50 \%)$ & $6(30 \%)$ \\
\hline
\end{tabular}

Table 2. Age, mean follow-up period, VAS of radicular leg pain, back pain and sensory dysesthesia, motor deficit before and after operations between the far lateral disc herniations and the paramedian disc herniations

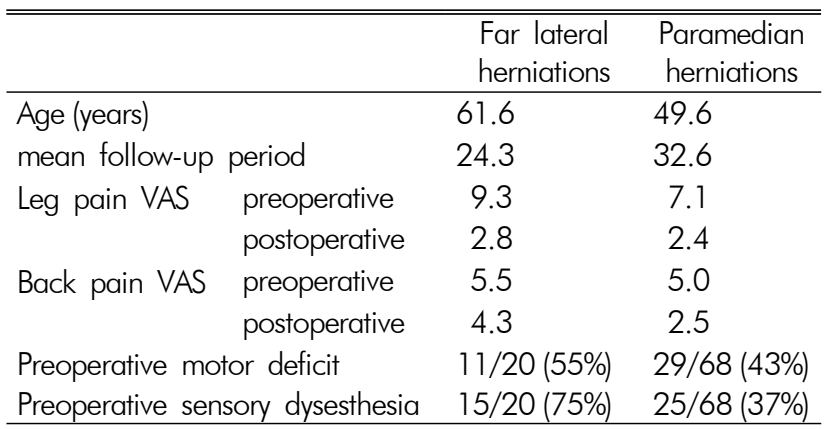

operation records.

In the far lateral disc herniation group, preoperatively, the average VAS of radicular leg pain was 9.3 and back pain was 5.5. After operation, the average VAS of radicular leg pain decreased to 2.8 and back pain decreased to 4.4. In the paramedian disc herniation group, preoperatively, the average VAS of radicular leg pain was 7.1 and back pain was 5. After operation, the average VAS of radicular leg pain decreased to 2.4 and back pain decreased to 2.5. Preoperatively, 11 (55\%) patients had motor deficit and $15(75 \%)$ patients had sensory dysethesia in the far lateral disc herniation group. Whereas, 29 (43\%) patients had motor deficit and 25 (37\%) patients had sensory dysethesia in the paramedian disc herniation group (Table 2).

Preoperatively, the far lateral disc herniation group had more severe radicular leg pain and showed more common sensory dysesthesia than the paramedian disc herniation group. They were proven to have statistically significant differences $(p<0.05)$. The occurrence of motor deficit was not statistically significant between two groups ( $p>0.05$ ).

In the far lateral disc herniation group, preoperatively, 15 patients (75\%) had sensory dysesthesia and among them, 4 patients (27\%) showed improvement. In the paramedian disc herniation group, preoperatively, 25 patients (37\%) had sensory dysesthesia and among them, 21 patients (84\%) showed improvement. The degree of improvement in sensory dysesthesia was statistically higher in the paramedian disc herniation group $(\mathrm{p}<0.05)$ (Table 3$)$.

In the far lateral disc herniation group, preoperatively, 11 patients (55\%) had motor deficit and among them, 10 patients (91\%) showed improvement. In the paramedian disc herniation group, preoperatively, 29 patients (43\%) had motor deficit and among them, 25 patients (86\%) showed improvement. The degree of improvement in motor deficit was not statistically significant between groups $(\mathrm{p}>0.05)$ (Table 4).

Table 3. Improvement of sensory dysesthesia after operation between the far lateral disc herniations and the paramedian disc herniations

\begin{tabular}{lcc}
\hline \hline Sensory dysesthesia & Paramedian disc & Far lateral disc \\
\hline Improved & $21(84 \%)$ & $4(26.7 \%)$ \\
Not improved & $4(16 \%)$ & $11(73.3 \%)$ \\
Total & 25 & 15 \\
\hline
\end{tabular}

Table 4. Improvement of motor deficit after operation between the far lateral disc herniations and the paramedian disc herniations

\begin{tabular}{lcc}
\hline \hline Motor deficit & Paramedian disc & Far lateral disc \\
\hline Improved & $25(86.2 \%)$ & $10(90.9 \%)$ \\
Not improved & $4(13.8 \%)$ & $1(9.1 \%)$ \\
Total & 29 & 11 \\
\hline
\end{tabular}




\section{DISCUSSION}

In 1974, Abdullah et al. first reported the clinical characteristics of far lateral herniated discs that occur far enough laterally (beneath or beyond facet), which distinguish them from the typical paramedian herniated discs that occur medial to the facet ${ }^{1)}$. At that time, the far lateral lumbar disc herniations were less familiar to neurosurgeons who could not demonstrate using myelography or due to limited exploration. But, since the introduction of CT and MRI, the foraminal or extraforaminal far lateral disc herniations had become a familiar disease to neurosurgeons ${ }^{10)}$.

According to previous studies, they suggested that the far lateral lumbar disc herniations showed poorer prognosis than the paramedian lumbar disc herniations. Several factors contribute to the poor prognosis of far lateral lumbar disc herniations. First, the diagnosis of far lateral disc herniations is difficult due to low incidence of the disease and it is easily overlooked on radiographic studies which lead to failure of treatment and poor outcome ${ }^{9,14)}$. Second, the far lateral lumbar disc herniations are frequently associated with additional pathological lesions within the vertebral canal at the same disc level (so called "double herniations"), 2,13,14) and this phenomenon causes confusion in distinguishing the exact pathological lesion that provokes the symptoms. The patients with double herniations are almost three times more likely to have residual sensory dysesthesia. Finally, the average age of far lateral disc herniations is higher than that of paramedian disc herniations and the overall outcome in the far lateral disc herniation group might be influenced by age factor ${ }^{11)}$. Our study also demonstrated that the far lateral disc herniation group was older than the paramedian disc herniation group.

Generally, the far lateral disc herniations have been known that they locate higher lumbar levels compared to the paramedian disc herniations ${ }^{4}$. Our analysis also demonstrated that the far lateral disc herniation occurs more frequently in upper lumbar levels compared to the paramedian disc herniation ( 25 $\%$ versus 3\%). Therefore it is important to have doubt about far lateral herniated disc if patients are elderly age and have severe radiating leg pain, higher lumbar levels symptoms and positive femoral nerve stretch test and we have experienced that sagittal MRI views through the neural foramen help demonstrate the far lateral herniated disc. As mentioned above, surgery for far lateral disc herniations seem to be associated a less favorable outcome but the results of this study are encouraging with respect to severe radiating leg pain improvement in surgical outcomes of far lateral disc herniations.

O'HARA et al. reported that the intensity of the radicular leg pain in far lateral prolapse is particularity severe due to direct contact of nuclear or annular fragments with the dorsal root ganglion" and also owing to the small size of the neural foramen, small far lateral herniated disc fragment can cause more severe symptoms ${ }^{8,13)}$. Our study also showed that the far lateral lumbar disc herniations presented more severe radicular leg pain than the paramedian lumbar disc herniations $(\mathrm{p}<0.05)$. This phenomenon is related to the shorter duration of symptoms before operation (64 days versus 131 days). Moreover, the patients in far lateral disc herniation group presented with extrusion disc which migrated superolaterally into the neural foramen, who showed the shorter duration of symptoms before operation than patients who presented with extrusion disc without migration (30 days versus 168 days) because we suggested migrated disc presented with more direct contact with the dorsal root ganglion.

In the previous studies, the far lateral disc herniations show less low back pain than the paramedian hernaitions ${ }^{1,5}$. To our knowledge, despite the paramedian muscle splitting approach and no facet joint violation during operation which seems like to have less low back pain, the far lateral disc herniation group presented with more severe low back pain than the paramedian disc herniation group in our study. Choi et al. reported that intervertebral disc and facet joint lesions are common finding with low back pain ${ }^{3)}$. In perspective of disc lesions, although discectomy increased in end plate degeneration and disc dehydration, it had only small contribution to back pain ${ }^{9}$. In perspective of the facet joint violation, Postacchini reported that there was no significant correlation between the mount of the facet joint removed and clinical result after $75 \%$ or less facetectomy ${ }^{12)}$. Our patients in far lateral disc herniation showed no facet joint violation during operation thus back pain was not caused by surgical technical factors such as the degree of facetectomy and so on. Thus, we guessed that the age factor contributed to more severe low back pain in far lateral disc herniation than paramedian disc herniation. In our study, the average age of far lateral disc herniation group was 61.6 year-old, while the average age of paramedian disc herniation group was 49.6 year-old. Therefore, considering the older age in the far lateral lumbar disc herniation group, we thought that it contributed to more severe low back pain, moreover the low back pain was not improved statistically significant in our study, but leg pain was improved statistically significant in the far lateral disc herniation group which means that far lateral herniated disc is less affected low back pain. Our study suggested that postoperative back pain was influenced by degenerative change associated with aging, regardless of surgical technique or etiology of disease in the far lateral disc herniations.

According to previous study, Porchet et al. reported that 
the clinical finding of a motor deficit was more reliable than sensory dysesthesia in far lateral disc herniations ${ }^{10)}$. Whereas Marquardt et al. reported that neurological deficits, if present, are parallel to those found in paramedian disc herniations ${ }^{4}$. But our study demonstrated that the sensory dysesthesia of far lateral disc herniations was more remarkable than the motor deficit (75\% versus 55\%). We suggest that less injury of the ventral root produces a less paresis of muscle innervated by the motor neuron in the far lateral disc herniations.

Our study showed that the far lateral lumbar disc herniation group showed less improvement of postoperative sensory dysesthesia than the paramedian lumbar disc herniation group $(p<0.05)$. As Rajaraman et al. suggested in far lateral lumbar disc herniations, postoperative severe burning dysesthesia are due to traction in the spinal nerve at the time of dissection ${ }^{15}$. Also, O'HARA suggested that early identification of the posterior ramus and safe dissection of the extraforaminal area reduce the risk of its avulsion from the dorsal root ganglion, which reduces the risk of postoperative severe sensory dysesthesia $^{7}$ so we tried to reduce the use of punch to prevent damage to the dorsal primary ramus by tearing off. This method reduced postoperative sensory dysesthesia. We thought that excessive manipulation of the root ganglion should be avoided during operation, because of its low sensitivity to thermal and mechanical injury.

\section{CONCLUSION}

Preoperatively, far lateral disc herniations had more severe radicular leg pain and common frequency of sensory dysesthesia. Postoperatively, the sensory dysesthesia was improved less and back pain was more severe in the far lateral disc herniations than in the paramedian disc herniations.

\section{REFERENCES}

1. Abdullah A, Ditto III EW, Byrd EB, Williams R: Extreme-lateral lumbar disc herniations: clinical syndrome and special problems of diagnosis. Journal of neurosurgery 41:229-234, 1974
2. Chang SB, Lee SH, Ahn Y, Kim JM: Risk factor for unsatisfactory outcome after lumbar foraminal and far lateral microdecompression. Spine (Phila Pa 1976) 31:1163-1167, 2006

3. Choi JW, Jang TW, Shin IY, Moon SM, Hwang HS, Jeong JH: Magnetic Resonance Imaging in Patients with Low Back Pain. Korean Journal of Spine 8:141-147, 2011

4. Epstein NE: Evaluation of varied surgical approaches used in the management of 170 far-lateral lumbar disc herniations: indications and results. J Neurosurg 83:648-656, 1995

5. Epstein NE: Foraminal and far lateral lumbar disc herniations: surgical alternatives and outcome measures. Spinal Cord 40: 491-500, 2002

6. Foley KT, Smith MM, Rampersaud YR: Microendoscopic approach to far-lateral lumbar disc herniation. Neurosurg Focus 7:e5, 1999

7. Maroon JC, Kopitnik TA, Schulhof LA, Abla A, Wilberger JE: Diagnosis and microsurgical approach to far-lateral disc herniation in the lumbar spine. J Neurosurg 72:378-382, 1990

8. Marquardt G, Bruder M, Theuss S, Setzer M, Seifert V: Ultralong-term outcome of surgically treated far-lateral, extraforaminal lumbar disc herniations: a single-center series. Eur Spine J 21:660-665, 2012

9. O'Hara LJ, Marshall RW: Far lateral lumbar disc herniation. The key to the intertransverse approach. J Bone Joint Surg Br 79:943-947, 1997

10. Porchet F, Fankhauser H, de Tribolet N: Extreme lateral lumbar disc herniation: clinical presentation in 178 patients. Acta Neurochir (Wien) 127:203-209, 1994

11. Porchet F, Chollet-Bornand A, de Tribolet N: Long-term follow up of patients surgically treated by the far-lateral approach for foraminal and extraforaminal lumbar disc herniations. J Neurosurg 90:59-66, 1999

12. Postacchini F, Cinotti G, Gumina S: Microsurgical excision of lateral lumbar disc herniation through an interlaminar approach. J Bone Joint Surg Br 80:201-207, 1998

13. Samini F, Bahadorkhan G, Ehsaei MR, Kheradmand H: Intraforaminal and extraforaminal far lateral lumbar disc herniation (a review of 63 cases). Medical Journal of The Islamic Republic of Iran (MJIRI) 22:63-67, 2008

14. Siebner HR, Faulhauer K: Frequency and specific surgical management of far lateral lumbar disc herniations. Acta Neurochir (Wien) 105:124-131, 1990

15. Viswanathan R, Swamy NK, Tobler WD, Greiner AL, Keller JT, Dunsker SB: Extraforaminal lumbar disc herniations: microsurgical anatomy and surgical approach. J Neurosurg 96:206211, 2002 